\title{
Non-steroidal anti-inflammatory drugs (NSAIDs) for acute sore throat (Protocol)
}

Venekamp RP, van Uum RT, Konstantinidis M, Lutje V, Schilder AGM, Hay AD, Damoiseaux RAMJ, Little $P$

Venekamp RP, van Uum RT, Konstantinidis M, Lutje V, Schilder AGM, Hay AD, Damoiseaux RAMJ, Little P. Non-steroidal anti-inflammatory drugs (NSAIDs) for acute sore throat (Protocol).

Cochrane Database of Systematic Reviews 2020, Issue 8. Art. No.: CD013709. DOI: 10.1002/14651858.CD013709.

www.cochranelibrary.com 
TABLE OF CONTENTS

HEADER 1

ABSTRACT

BACKGROUND

OBJECTIVES

METHODS

ACKNOWLEDGEMENTS

REFERENCES

ADDITIONAL TABLES

APPENDICES

HISTORY

CONTRIBUTIONS OF AUTHORS

DECLARATIONS OF INTEREST 
[Intervention Protocol]

\section{Non-steroidal anti-inflammatory drugs (NSAIDs) for acute sore throat}

Roderick P Venekamp¹, Rick T van Uum¹, Menelaos Konstantinidis², Vittoria Lutje ${ }^{3}$, Anne GM Schilder ${ }^{4}$, Alastair D Hay ${ }^{5}$, Roger AMJ Damoiseaux ${ }^{1}$, Paul Little 6

1Julius Center for Health Sciences and Primary Care, University Medical Center Utrecht, Utrecht University, Utrecht, Netherlands. 2Department of Mathematics and Statistics, York University, Toronto, Canada. ${ }^{3}$ Cochrane Infectious Diseases Group, Liverpool School of Tropical Medicine, Liverpool, UK. ${ }^{4}$ evidENT, Ear Institute, University College London, London, UK. ${ }^{5}$ Centre for Academic Primary Care, NIHR School for Primary Care Research, Bristol Medical School: Population Health Sciences, University of Bristol, Bristol, UK. ${ }^{6}$ Primary Care and Population Sciences, Faculty of Medicine, Aldermoor Health Centre, University of Southampton, Southampton, UK

Contact address: Roderick P Venekamp, r.p.venekamp@umcutrecht.nl.

Editorial group: Cochrane Acute Respiratory Infections Group.

Publication status and date: New, published in Issue 8, 2020.

Citation: Venekamp RP, van Uum RT, Konstantinidis M, Lutje V, Schilder AGM, Hay AD, Damoiseaux RAMJ, Little P. Non-steroidal antiinflammatory drugs (NSAIDs) for acute sore throat (Protocol). Cochrane Database of Systematic Reviews 2020, Issue 8. Art. No.: CD013709. DOI: 10.1002/14651858.CD013709.

Copyright () 2020 The Cochrane Collaboration. Published by John Wiley \& Sons, Ltd.

\section{A B S T R A C T}

\section{Objectives}

This is a protocol for a Cochrane Review (intervention). The objectives are as follows:

To assess the effects of non-steroidal anti-inflammatory drugs (NSAIDs) for acute sore throat in children and adults. 


\section{B A C K G R O U N D}

\section{Description of the condition}

Acute sore throat, including pharyngitis and tonsillitis, is a very common condition: the community incidence has been estimated at 1.57 per person-year in a recent UK-based population-based cohort study (Mehta 2017). With around 1 in 10 people seeking medical care (Mehta 2017), sore throat is a common reason for ambulatory care visits in high-income countries (Fleming-Dutra 2016; Gulliford 2014; Lewnard 2020). The condition can be of viral or bacterial origin. The most prominent bacterium is group $A$ beta-haemolytic streptococcus (GAS), which is isolated in around $10 \%$ to $15 \%$ of adult and $15 \%$ to $30 \%$ of childhood cases in Western countries (Bisno 2001; Hayward 2017; Linder 2005), but epidemiology and severity may vary depending on geographical location, with a GAS-positive rate of $42 \%$ reported in children with sore throat living in a low-income setting (DeWyer 2020).

Irrespective of causative pathogen (i.e. whether viral or bacterial), there is a need for symptom relief. High-quality evidence indicates that antibiotics provide only marginal benefits in alleviating symptoms in otherwise healthy people with uncomplicated sore throat living in high-income countries (Spinks 2013), and the number needed to treat to prevent serious suppurative complications such as peritonsillar abscess is estimated at over 4000 people in these countries (Petersen 2007). Nevertheless, antibiotic prescribing for people with this condition remains at a high rate (Gulliford 2014; van den Broek d'Obrenan 2014). Importantly, sore throat is amongst the conditions with the highest antibiotic overprescribing rates (Dekker 2015; Fleming-Dutra 2016), thereby exposing people to unnecessary side effects (Gillies 2015), and contributing to the emergence of antimicrobial resistance (Costelloe 2010).

\section{Description of the intervention}

Non-steroidal anti-inflammatory drugs (NSAIDs) inhibit prostaglandin synthesis by competitive binding to the cyclooxygenase (COX) enzymes (COX-1 or COX-2, or both) and can be divided into traditional (non-selective NSAIDs, which inhibit the activity of both COX-1 and COX-2) and selective COX-2 inhibitors (Bruno 2014). COX-2 inhibition leads to reduced release of pyrogenic molecules in the inflamed cells (mainly prostaglandin E2) causing the anti-inflammatory, antipyretic, and analgesic effects of NSAIDs (Bruno 2014; Rainsford 2009). COX-1 inhibition is mainly responsible for the gastrointestinal adverse effects of NSAIDs (Bruno 2014). NSAIDs, especially aspirin, also inhibit platelet aggregation due to the inability of the COX enzyme to synthesise thromboxane precursors within platelets.

A number of NSAIDs are on the market that are available overthe-counter (OTC) in most countries. The most well-known NSAIDs include aspirin, ibuprofen, diclofenac, and naproxen.

NSAIDs can be administered orally (suspension, tablets, capsules, lozenges (partially locally)), locally (e.g. sprays into the throat), rectally (suppositories), or by intramuscular or intravenous injection.

\section{How the intervention might work}

Theoretically, both positive and negative effects of NSAIDs used for acute sore throat can be anticipated. Inflammation and subsequent swelling of the oropharyngeal mucosa are thought to play a central role in the occurrence and maintenance of the most prominent symptoms of sore throat such as pain, difficulties swallowing, and ingesting food or drink. Reducing the host inflammatory response may therefore alleviate symptoms and expedite recovery. A recent Cochrane Review showed that corticosteroids in addition to antibiotics increase the likelihood of both resolution and improvement of pain in patients with sore throat (de Cassan 2020). The anti-inflammatory, antipyretic, and analgesic effects of NSAIDs could therefore also have a beneficial impact on sore throat symptoms. Conversely, NSAIDs may have a negative impact on the resolution of acute respiratory tract infections due to their interference with human neutrophil functioning (Kaplan 1984), which in turn might result in prolonged illness duration, an increase in doctor reconsultations, and an enhanced risk of suppurative complications including peritonsillar abscess (Basille 2017; Le Bourgeois 2016; Little 2013; Little 2016; Nicollas 2020; Piroulas 2019; Voiriot 2011).

\section{Why it is important to do this review}

NSAIDs are available over the counter in many countries and are commonly used to alleviate acute respiratory tract infection symptoms including those associated with sore throat such as pain and fever. However, their potential benefits for this condition are unclear and have not been recently systematically reviewed (Thomas 2000). Furthermore, any potential benefits should be carefully balanced against the risk of side effects. NSAIDs have long since been associated with well-known side effects such as gastrointestinal bleeding, renal failure, and cardiovascular diseases. More recently, observational and randomised controlled trial evidence has become available indicating that NSAIDs may cause direct harm on the progression of acute respiratory tract infections including an increased risk of prolonged illness duration, doctor reconsultations, and suppurative complications including peritonsillar abscess (Basille 2017; Le Bourgeois 2016; Little 2013; Little 2016; Nicollas 2020; Piroulas 2019; Voiriot 2011). As such, it is important to systematically synthesise the available evidence on the clinical benefit and adverse events of NSAIDs for sore throat in children and adults.

\section{O B JECTIVES}

To assess the effects of non-steroidal anti-inflammatory drugs (NSAIDs) for acute sore throat in children and adults.

\section{METHODS}

\section{Criteria for considering studies for this review}

\section{Types of studies}

We will include both individually randomised controlled trials (RCTs), that is where the participant is the unit of randomisation, and cluster-RCTs. In addition, we will include the following types of studies for the primary adverse event outcome 'infectionrelated adverse events, defined as reconsultation rates with the same symptoms (sore throat) or progressive/new symptoms, and/ or complications (e.g. peritonsillar abscess) within one month': quasi-RCT (Q-RCT), non-RCT (NRCT), prospective cohort study, and retrospective cohort study. We aim to include these types of observational studies for our primary adverse event outcome since RCTs are usually underpowered to identify potentially important differences in the occurrence of rare complications 
between treatment groups; this approach might therefore allow us to more carefully weigh benefits and harms of NSAIDs use in patients with acute sore throat.

We will include studies irrespective of publication status, date of publication, or language.

\section{Types of participants}

We will include both children and adults presenting in a primary healthcare setting (i.e. patients who are not referred by a physician because of the current sore throat episode, or patients presenting through self-referral in ambulatory care or an emergency department) with sore throat. We will include the following conditions:

1. clinical signs and symptoms suggestive of acute tonsillitis inflammation of the tonsils;

2. clinical signs and symptoms suggestive of pharyngitis inflammation of the oropharynx; and

3. clinical syndrome of sore throat (painful throat, odynophagia).

We will exclude studies of referred patients, hospitalised patients, as well as those involving highly specialised populations (e.g. those with glandular fever, immunodeficiency, peritonsillar abscess, or sore throat following tonsillectomy).

\section{Types of interventions}

We will include studies irrespective of the dose, duration, or method of administration of NSAIDs.

We will include trials comparing:

1. NSAIDs with placebo;

2. NSAIDs with no treatment;

3. NSAIDs with paracetamol (acetaminophen);

4. NSAID and paracetamol (acetaminophen) with placebo and paracetamol (acetaminophen);

5. NSAIDs and paracetamol (acetaminophen) with paracetamol (acetaminophen) alone;

6. NSAIDs with other pharmacological treatments such as lozenges (not containing NSAIDs) and herbal medicinal products; and

7. NSAIDs with non-pharmacological treatments such as physical cooling (e.g. ice cubes, wet cloths) and topical treatments (e.g. gargling with povidone-iodine or salt).

We will include trials reporting combined interventions if they allow a direct comparison between the NSAID and control groups (e.g. coadministration of antibiotics in both groups). We will address the following comparison pairs separately:

1. NSAIDs and antibiotics versus placebo and antibiotics; and

2. NSAIDs and antibiotics versus antibiotics alone.

\section{Types of outcome measures}

We will analyse the following outcomes in the review, but will not use them as a basis for including or excluding studies.

\section{Primary outcomes}

1. Complete resolution of pain at two to three days ( 48 to 72 hours; when symptoms are usually worst).
2. Infection-related adverse events, defined as reconsultation with the same symptoms (sore throat) or progressive/new symptoms, and/or complications (e.g. peritonsillar abscess) within one month.

\section{Secondary outcomes}

1. Disease-specific quality of life using a validated instrument.

2. Complete resolution of pain at very short periods of time ( 1 hour, 2 to 3 hours, 4 to 6 hours).

3. Complete resolution of pain at various other time points (24 hours, 4 to 7 days).

4. Mean time to complete resolution of pain.

5. Pain intensity at various time points ( 1 hour, 2 to 3 hours, 4 to 6 hours, 24 hours, 2 to 3 days, and 4 to 7 days).

6. Mean time to resolution of fever.

7. Mean time to complete resolution of total symptoms (recovery).

8. Mean time to return to a normal diet.

9. Sore throat recurrences after one month.

10.Adverse effects likely to be related to the use of NSAIDs: i) proportion of participants with gastrointestinal (GI) complaints (excluding GI bleeding); and ii) proportion of participants with GI bleeding, and/or renal failure, and/or cardiovascular diseases (myocardial infarction; transient ischaemic attack; cerebrovascular accident).

\section{Search methods for identification of studies}

We will conduct systematic searches for RCTs and controlled clinical trials. We will perform a separate search for non-randomised studies reporting on infection-related adverse events, using established filters to identify reports of adverse events. We will attempt to identify all relevant studies regardless of language or publication status (published, unpublished, in press, ongoing).

\section{Electronic searches}

We will search the following databases from inception to present, using the search terms and strategy described in Appendix 1:

1. Cochrane Central Register of Controlled Trials (CENTRAL), published in the Cochrane Library;

2. MEDLINE (Ovid);

3. Embase (Ovid); and

4. CINAHL (Cumulative Index to Nursing and Allied Health Literature) (EBSCOHost).

We will also search the World Health Organization (WHO) International Clinical Trials Registry Platform (www.who.int/ictrp/ en/) and US National Institutes of Health Ongoing Trials Register ClinicalTrials.gov (clinicaltrials.gov) for trials in progress. We will also run searches of Google Scholar using the same terms to retrieve grey literature and other trials. We will report all search details in the final version of the review.

\section{Searching other resources}

We may contact original authors for clarification and further data if trial reports are unclear. We will arrange translations of papers where necessary.

We will also check the reference lists of all included studies and those of relevant review articles identified by the searches. 


\section{Data collection and analysis}

\section{Selection of studies}

Two review authors (RPV, RTVU) will independently screen titles and abstracts of the records obtained from the searches. We will first identify and exclude duplicates, and then screen the titles and abstracts of the unique records to identify potentially relevant studies. We will retrieve the full-text study reports/publications of all potentially eligible studies. The same two review authors will independently review the full texts against the predefined inclusion and exclusion criteria for eligibility. Any disagreements will be resolved through discussion or by consulting a third review author $(\mathrm{PL})$ if required.

We will collate multiple reports of the same study so that each study, rather than each report, is the unit of interest in the review. We will record the selection process in sufficient detail to complete a PRISMA flow diagram and 'Characteristics of excluded studies' table (Moher 2009).

\section{Data extraction and management}

We will use a data collection form for the extraction of study characteristics and outcome data that has been piloted on at least one study in the review. Two review authors (RPV, RTVU) will independently extract the following study characteristics from the included studies:

1. methods: study design, total duration of study, details of any 'run-in' period, number of study centres and location, study setting, withdrawals, and date of study;

2. participants: number, mean age, age range, gender, severity of condition, diagnostic criteria, inclusion criteria, and exclusion criteria;

3. interventions: intervention (including instruction about NSAIDs use and NSAID type), comparison, concomitant medications, and excluded medications;

4. outcomes: primary and secondary outcomes specified and collected, and time points reported; and

5. notes: funding for trial, and notable conflicts of interest of trial authors.

Two review authors (RPV, RTVU) will independently extract outcome data from the included studies. We will note in the 'Characteristics of included studies' table (see Table 1 for template) if outcome data are not reported in a useable way. Any disagreements will be resolved by consensus or by involving a third review author (PL). One review author (RPV) will transfer data into the Review Manager file using RevMan Web (RevMan Web 2019). We will double-check that data are entered correctly by comparing the data presented in the systematic review with the study reports. A second review author (RTvU) will spot-check study characteristics for accuracy against the trial report.

\section{Assessment of risk of bias in included studies}

Two review authors (RPV, RTVU) will independently assess risk of bias for the included RCTs using the Cochrane 'Risk of bias' tool for randomised trials (RoB 2) (Sterne 2019), according to the guidance in the latest version of the Cochrane Handbook for Systematic Reviews of Interventions (Higgins 2019). Any disagreements will be resolved by discussion or by involving another review author (PL).
We will assess risk of bias as 'low', 'high', or 'some concerns' for each of the following five domains:

1. bias arising from the randomisation process;

2. bias due to deviations from the intended interventions;

3. bias due to missing outcome data;

4. bias in measurement of outcome; and

5. bias in selection of the reported results.

For cluster-RCTs, we will assess risk of bias using these five domains in addition to a sixth domain described in the latest version of the Cochrane Handbook for Systematic Reviews of Interventions (Higgins 2019), namely "Bias arising from the timing of identification and recruitment of participants".

We will provide a quote from the study report together with a justification for our judgement in the 'Risk of bias' table. We will summarise the 'Risk of bias' judgements across different studies for each of the domains listed. Where information on risk of bias relates to unpublished data or correspondence with an individual trial author, we will note this in the 'Risk of bias' table.

We will manage the risk of bias through the Microsoft Excel tool available at www.riskofbias.info (Sterne 2019). For assessment of the overall risk of bias, we will use signalling questions and algorithm tools (Higgins 2019). We will assess risk of bias of the RCTs using the RoB 2 tool based on the following outcomes:

1. complete resolution of pain at two to three days ( 48 to 72 hours; when symptoms are usually worst);

2. infection-related adverse events, defined as reconsultation with the same symptoms (sore throat) or progressive/new symptoms, and/or complications (e.g. peritonsillar abscess) within one month;

3. complete resolution of pain at various other time points (24 hours, four to seven days);

4. mean time to resolution of fever;

5. mean time to complete resolution of total symptoms (recovery);

6. mean time to return to normal diet; and

7. adverse effects likely to be related to the use of NSAIDs.

We will store the consensus decisions for the signalling questions as supplemental data.

The effect of interest will be 'effect of assignment' (intention-totreat (ITT) principle).

We will assess risk of bias for relevant non-randomised studies using the ROBINS-I tool (Sterne 2016). We will use this tool to assess the risk of bias of the primary adverse event outcome 'infection-related adverse events, defined as reconsultation rates with the same symptoms (sore throat) or progressive/new symptoms, and/or complications (e.g. peritonsillar abscess) within one month'. We will assess the following confounders: age, comorbidity, aetiology, fever, symptom severity, prior duration of symptoms, exudate, severe inflammation of the pharynx, and cotreatment with antibiotics. 


\section{Assessment of bias in conducting the systematic review}

We will conduct the review according to this published protocol and report any deviations from it in the 'Differences between protocol and review' section of the systematic review.

\section{Measures of treatment effect}

We will enter the outcome data for each study into the data tables in RevMan Web to calculate the treatment effects (RevMan Web 2019). We will use risk ratios (RR) with $95 \%$ confidence intervals (Cls) for dichotomous outcomes and mean differences (MD) with standard deviations (SD) or as standardised mean differences (SMD) for continuous outcomes. Where SMD has been computed, we will reexpress the outcomes in a single natural scale (to be determined based on the scales from the observed studies).

For each of the key outcomes listed in the 'Summary of findings' table, we will present the results for the absolute numbers (in addition to the relative numbers) based on the pooled results and compared to the assumed risk. Where applicable, we will also calculate the number needed to treat for an additional beneficial outcome (NNTB) and number needed to treat for an additional harmful outcome (NNTH) using the pooled results.

\section{Unit of analysis issues}

For cluster-RCTs, we will consider potential differences between the intervention effects being estimated and check whether clustering was taken into account in the analysis of the individual trials. Additionally, as per the Cochrane Handbook for Systematic Reviews of Interventions (Higgins 2019), we will report on whether or not the sample sizes were estimated through the intracluster/intraclass correlation coefficient (ICC) and whether the analyses were carried out at the cluster level. Where trials take multiple measurements or observations of a single outcome in the same participants (repeated measurements), we will only extract and analyse the datapoints consistent with the times of follow-up defined in the primary and secondary outcomes in this protocol.

\section{Dealing with missing data}

We will contact individual trial authors or study sponsors to verify key study characteristics and to obtain missing numerical outcome data where possible (e.g. when a study is identified as abstract only). Where this is not possible, and the missing data are thought to introduce serious bias, we will explore the impact of including such studies in the overall assessment of results by a sensitivity analysis, provided sufficient data are available.

If SDs are missing and they cannot be obtained from the trial authors, we will approximate them using other available statistics such as $P$ values or alternative methods according to the methods described in the Cochrane Handbook for Systematic Reviews of Interventions (Higgins 2019).

Apart from imputations for missing SDs, we will not conduct any other imputations. We will extract and analyse all data using the available-case analysis method based on the ITT principle, that is by analysing all participants in the groups to which they had originally been randomised.

\section{Assessment of heterogeneity}

We will assess clinical heterogeneity by reviewing the included trials for potential differences between trials in study populations, interventions or comparisons used, and outcomes measured.

We will assess statistical heterogeneity for each outcome by visually inspecting the forest plots and by using the $\mathrm{I}^{2}$ statistic, with investigation of $\mathrm{I}^{2}$ values over the ranges described in the Cochrane Handbook for Systematic Reviews of Interventions (Higgins 2019).

\section{Assessment of reporting biases}

To assess outcome reporting bias, we will search the internet and ClinicalTrials.gov (clinicaltrials.gov) for available study protocols to determine whether the outcomes reported in the included trials were predefined and whether all outcomes listed in the study protocol are reported in the publication. We propose that if there are sufficient trials, we will assess reporting bias by using funnel plots. If we are able to pool more than 10 trials, we will create and examine a funnel plot to explore possible small-study and publication biases.

\section{Data synthesis}

We will analyse the data according to the ITT principle. Where data are missing or there was loss to follow-up, we will conduct an available-case analysis.

We will pool data from studies we judge to be clinically homogeneous using RevMan Web (RevMan Web 2019). If more than one study provides useable data in any single comparison, we will perform a meta-analysis using a random-effects (DerSimonian and Laird) model to provide a more conservative estimate. We will include all eligible studies in the primary analyses, but we will perform separate meta-analyses for RCTs and non-RCTs (for the primary adverse event outcome).

For non-RCTs, our primary analyses will include eligible studies where the data have been adjusted (with common criteria). Secondary analyses will consist of combining eligible studies where the data have not been adjusted. We will discuss the limitations of these analyses in both cases.

\section{Subgroup analysis and investigation of heterogeneity}

We will perform subgroup analyses planned a priori for the following characteristics if there are at least 10 studies available for each subgroup stratum:

1. age (children versus adults);

2. Centor criteria (0 to 2 versus 3 to 4 );

3. prior duration (three days or less versus over three days);

4. fever (body temperature $>38^{\circ} \mathrm{C}$ present versus absent);

5. NSAID type (sprays versus lozenges versus tablets/capsules/ suppositories/injections); and

6. instruction about NSAIDs use (at regular time intervals versus as needed).

We will use the $\mathrm{Chi}^{2}$ test to test for subgroup interactions in Review Manager (RevMan Web 2019). 


\section{Sensitivity analysis}

We plan to carry out sensitivity analyses to determine the robustness of our 'Risk of bias' findings for the included studies by excluding studies with high risk of overall bias for the results as assessed using the Cochrane RoB 2 tool (Higgins 2019).

\section{Summary of findings and assessment of the certainty of the evidence}

We will create a 'Summary of findings' table using the following seven top-priority outcomes:

1. complete resolution of pain at two to three days ( 48 to 72 hours; when symptoms are usually worst);

2. infection-related adverse events, defined as reconsultation with the same symptoms (sore throat) or progressive/new symptoms, and/or complications (e.g. peritonsillar abscess) within one month;

3. complete resolution of pain at various other time points (24 hours, four to seven days);

4. mean time to resolution of fever;
5. mean time to complete resolution of total symptoms (recovery);

6. mean time to return to normal diet; and

7. adverse effects likely to be related to the use of NSAIDs.

We will use the five GRADE considerations (study limitations, inconsistency, imprecision, indirectness, and publication bias) to rate the overall certainty of the evidence for each outcome (Atkins 2004). We will use the methods and recommendations described in the Cochrane Handbook for Systematic Reviews of Interventions (Higgins 2019), employing GRADEpro GDT software (GRADEpro GDT). We will justify all decisions to down- or upgrade the certainty of the evidence using footnotes, and will make comments to aid the reader's understanding of the review where necessary.

\section{ACK N O WLEDGEMENTS}

The methods section of this protocol is based on a standard template developed by the Cochrane Airways Group and adapted by the Cochrane Acute Respiratory Infections Group. We wish to thank the following people for commenting on the draft protocol: Amanda Roberts, Ann Fonfa, Mark Ebell, Anneliese Spinks, Robert Ware, and Johannes $C$ van der Wouden. 


\section{RE F E R E N C E S}

\section{Additional references}

\section{Atkins 2004}

Atkins D, Best D, Briss PA, Eccles M, Falck-Ytter Y, Flottorp S, et al, GRADE Working Group. Grading quality of evidence and strength of recommendations. BMJ 2004;328(7454):1490.

\section{Basille 2017}

Basille D, Plouvier N, Trouve C, Duhaut P, Andrejak C, Jounieaux V. Non-steroidal anti-inflammatory drugs may worsen the course of community-acquired pneumonia: a cohort study. Lung 2017;195:201-8.

\section{Bisno 2001}

Bisno AL. Acute pharyngitis. New England Journal of Medicine 2001;344(3):205-11.

\section{Bruno 2014}

Bruno A, Tacconelli S, Patrignani P. Variability in the response to non-steroidal anti-inflammatory drugs: mechanisms and perspectives. Basic and Clinical Pharmacology and Toxicology 2014;114(1):56-63.

\section{Costelloe 2010}

Costelloe C, Metcalfe C, Lovering A, Mant D, Hay AD. Effect of antibiotic prescribing in primary care on antimicrobial resistance in individual patients: systematic review and metaanalysis. BMJ 2010;340:c2096.

\section{de Cassan 2020}

de Cassan S, Thompson MJ, Perera R, Glasziou PP, Del Mar CB, Heneghan CJ, et al. Corticosteroids as standalone or add-on treatment for sore throat. Cochrane Database of Systematic Reviews 2020, Issue 5. Art. No: CD008268. [DOI: 10.1002/14651858.CD008268.pub3]

\section{Dekker 2015}

Dekker AR, Verheij T, van der Velden AW. Inappropriate antibiotic prescription for respiratory tract indications: most prominent in adult patients. Family Practice 2015;32(4):401-7.

\section{DeWyer 2020}

DeWyer A, Scheel A, Webel AR, Longenecker CT, Kamarembo J, Aliku T, et al. Prevalence of group A $\beta$-hemolytic streptococcal throat carriage and prospective pilot surveillance of streptococcal sore throat in Ugandan school children. International Journal of Infectious Diseases 2020;93:245-51.

\section{Fleming-Dutra 2016}

Fleming-Dutra KE, Hersh AL, Shapiro DJ, Bartoces M, Enns EA, File TM Jr, et al. Prevalence of inappropriate antibiotic prescriptions among US ambulatory care visits, 2010-2011. JAMA 2016;315(17):1864-73.

\section{Gillies 2015}

Gillies M, Ranakusuma A, Hoffmann T, Thorning S, McGuire T, Glasziou P, et al. Common harms from amoxicillin: a systematic review and meta-analysis of randomized placebo-controlled trials for any indication. Canadian Medical Association Journal 2015;187(1):E21-31.

\section{GRADEpro GDT [Computer program]}

McMaster University (developed by Evidence Prime) GRADEpro GDT. Hamilton (ON): McMaster University (developed by Evidence Prime), accessed 12 February 2020. Available at gradepro.org.

\section{Gulliford 2014}

Gulliford MC, Dregan A, Moore MV, Ashworth M, Staa TV, McCann G, et al. Continued high rates of antibiotic prescribing to adults with respiratory tract infection: survey of $568 \mathrm{UK}$ general practices. BMJ Open 2014;4(10):e006245.

\section{Hayward 2017}

Hayward GN, Hay AD, Moore MV, Jawad S, Williams N, Voysey M, et al. Effect of oral dexamethasone without immediate antibiotics vs placebo on acute sore throat in adults: a randomized clinical trial. JAMA 2017;317(15):1535-43.

\section{Higgins 2019}

Higgins JPT, Thomas J, Chandler J, Cumpston M, Li T, Page MJ, Welch VA (editors). Cochrane Handbook for Systematic Reviews of Interventions version 6.0 (updated July 2019). Cochrane, 2019. Available from www.training.cochrane.org/handbook.

\section{Kaplan 1984}

Kaplan HB. Effects of non-steroidal anti-inflammatory agents on human neutrophil functions in vitro and in vivo. Biochemical Pharmacology 1984;33(3):371-8.

\section{Le Bourgeois 2016}

Le Bourgeois M, Ferroni A, Leruez-Ville M, Varon E, Thumerelle C, Brémont $F$, et al. Nonsteroidal anti-inflammatory drug without antibiotics for acute viral infection increases the empyema risk in children: a matched case-control study. Journal of Pediatrics 2016;175:47-53.

\section{Lefebvre 2019}

Lefebvre C, Glanville J, Briscoe S, Littlewood A, Marshall C, Metzendorf $\mathrm{M}-\mathrm{I}$, et al. Chapter 4: Searching for and selecting studies. In: Higgins JPT, Thomas J, Chandler J, Cumpston M, Li T, Page MJ, Welch VA (editors). Cochrane Handbook for Systematic Reviews of Interventions version 6.0 (updated July 2019). Cochrane, 2019. Available from www.training.cochrane.org/handbook.

\section{Lewnard 2020}

Lewnard JA, King LM, Fleming-Dutra KE, Link-Gelles R, Van Beneden CA. Incidence of pharyngitis, sinusitis, acute otitis media, and outpatient antibiotic prescribing preventable by vaccination against group A Streptococcus in the United States. Clinical Infectious Diseases 2020; May 6:Online ahead of print. [DOI: 10.1093/cid/ciaa529] 


\section{Linder 2005}

Linder JA, Bates DW, Lee GM, Finkelstein JA. Antibiotic treatment of children with sore throat. JAMA 2005;294(18):2315-22.

\section{Little 2013}

Little P, Moore M, Kelly J, Williamson I, Leydon G, McDermott L, et al. Ibuprofen, paracetamol, and steam for patients with respiratory tract infections in primary care: pragmatic randomised factorial trial. BMJ 2013;347:f6041. [DOI: 10.1136/ bmj.f6041]

\section{Little 2016}

Little P, Stuart B, Andreou P, McDermott L, Joseph J, Mullee M, et al. Primary care randomised controlled trial of a tailored interactive website for the self-management of respiratory infections (Internet Doctor). BMJ Open 2016;6(4):e009769. [DOI: 10.1136/bmjopen-2015-009769]

\section{Mehta 2017}

Mehta N, Schilder A, Fragaszy E, Evans HER, Dukes O, Manikam $L$, et al. Antibiotic prescribing in patients with selfreported sore throat. Journal of Antimicrobial Chemotherapy 2017;72(3):914-22.

\section{Moher 2009}

Moher D, Liberati A, Tetzlaff J, Altman DG, The PRISMA Group. Preferred reporting items for systematic reviews and metaanalyses: The PRISMA Statement. BMJ 2009;339:2535.

\section{Nicollas 2020}

Nicollas R, Moreddu E, Le Treut-Gay C, Mancini J, Akkari M, Mondain M, et al. Ibuprofen as risk-factor for complications of acute anterior sinusitis in children. European Annals of Otorhinolaryngology, Head and Neck Disease 2020;137(2):99-103.

\section{Petersen 2007}

Petersen I, Johnson AM, Islam A, Duckworth G, Livermore DM, Hayward AC. Protective effect of antibiotics against serious complications of common respiratory tract infections: retrospective cohort study with the UK General Practice Research Database. BMJ 2007;335(7627):982.

\section{Piroulas 2019}

Piroulas C, Devillers L, Souty C, Sicsic J, Boisnault P, François M. Non-steroids anti-inflammatory drugs and risk of peritonsillar abscess in pharyngitis: a French longitudinal study in primary care. Family Practice 2019;36(4):425-30.

\section{Rainsford 2009}

Rainsford KD. Ibuprofen: pharmacology, efficacy and safety. Inflammopharmacology 2009;17(6):275-342.

\section{RevMan Web 2019 [Computer program]}

The Cochrane Collaboration Review Manager Web (RevMan Web). The Cochrane Collaboration, 2019. Available at: revman.cochrane.org.

\section{Spinks 2013}

Spinks A, Glasziou PP, Del Mar CB. Antibiotics for sore throat. Cochrane Database of Systematic Reviews 2013, Issue 11. Art. No: CD000023. [DOI: 10.1002/14651858.CD000023.pub4]

\section{Sterne 2016}

Sterne JAC, Hernán MA, Reeves BC, Savović J, Berkman ND, Viswanathan M, et al. ROBINS-I: a tool for assessing risk of bias in non-randomized studies of interventions. BMJ 2016;355:i4919.

\section{Sterne 2019}

Sterne JAC, Savović J, Page MJ, Elbers RG, Blencowe NS, Boutron I, et al. RoB 2: a revised tool for assessing risk of bias in randomised trials. BMJ 2019;366:14898.

\section{Thomas 2000}

Thomas M, Del Mar C, Glasziou P. How effective are treatments other than antibiotics for acute sore throat? British Journal of General Practice 2000;50(459):817-20.

\section{van den Broek d'Obrenan 2014}

van den Broek d'Obrenan J, Verheij TJ, Numans ME, van der Velden AW. Antibiotic use in Dutch primary care: relation between diagnosis, consultation and treatment. Journal of Antimicrobial Chemotherapy 2014;69(6):1701-7.

\section{Voiriot 2011}

Voiriot G, Dury S, Parrot A, Mayaud C, Fartoukh M. Nonsteroidal antiinflammatory drugs may affect the presentation and course of community-acquired pneumonia. Chest 2011;139(2):387-94.

\section{ADDITIONAL TABLES}

\section{Table 1. 'Characteristics of included studies' table template}

\section{Methods}

Study design (e.g. parallel RCT, cross-over RCT, cluster-RCT)

Study duration: date of first recruitment to last follow-up

\section{Participants}


Table 1. 'Characteristics of included studies' table template (Continued)

Control group:

Sex (M/F): treatment (N/N M/F); control (N/N M/F)

Any other relevant info, such as comorbidities

Exclusion criteria

$<$ list>

$\begin{array}{ll}\text { Interventions } & \text { Treatment group } \\ \text { Intervention } \\ \text { Dose, duration, frequency, administration } \\ \text { Other relevant info } \\ \text { Control group } \\ \text { Intervention (e.g. placebo, no treatment) } \\ \text { Dose, duration, frequency, administration } \\ \text { Other relevant info }\end{array}$

Outcomes

Primary outcomes

$<$ list $>$

\section{Secondary outcomes}

$<$ list>

Note: describe the methods used to measure the outcomes

\section{Notes}

Declaration of Interest:

Funding source:

Contact with study authors for additional information:

Other:

\section{APPENDICES}

\section{Appendix 1. Detailed search strategy}

Ovid MEDLINE ${ }^{\circledR}$ and Epub Ahead of Print, In-Process \& Other Non-Indexed Citations, Daily and Versions ${ }^{\circledast} 1946$ to present

1 (sore adj2 throat $\left.^{\star}\right) \cdot \mathrm{mp}$.

2 Pharyngitis/

3 pharyngit*.mp.

4 Nasopharyngitis/

5 nasopharyngit*.mp.

6 rhinopharyngitis.mp.

7 Tonsillitis/

8 Tonsillit*.mp.

9 (tonsil $^{\star}$ adj2 (inflam* ${ }^{\star}$ infect $\left.\left.{ }^{\star}\right)\right) \cdot m p$.

10 ((throat* or pharyn*) adj3 (infect* or inflam*)).mp.

111 or 2 or 3 or 4 or 5 or 6 or 7 or 8 or 9 or 10

12 Anti-Inflammatory Agents, Non-Steroidal/ or non-steroidal antiinflammatory agent*.mp.

$13 \mathrm{NSAID}^{\star} . \mathrm{mp}$. 
14 ((non-steroid ${ }^{\star}$ or nonsteroid* or non steroid $\left.{ }^{\star}\right)$ and (anti-inflammator ${ }^{\star}$ or antiinflammator $^{\star}$ or anti inflammator $\left.\left.{ }^{\star}\right)\right)$.mp.

15 apazone.mp. or Apazone/

16 aspirin.mp. or Aspirin/

17 celecoxib.mp. or Celecoxib/

18 diclofenac.mp. or Diclofenac/

19 diflunisal.mp. or Diflunisal/

20 etodolac.mp. or Etodolac/

21 fenoprofen.mp. or Fenoprofen/

22 flurbiprofen.mp. or Flurbiprofen/

23 ibuprofen.mp. or Ibuprofen/

24 indomethacin.mp. or Indomethacin/

25 ketoprofen.mp. or Ketoprofen/

26 Ketorolac/ or Ketorolac Tromethamine/ or ketorolac.mp.

27 Meclofenamic Acid.mp. or Meclofenamic Acid/

28 meclofenamate.mp.

29 meloxicam.mp. or Meloxicam/

30 (methyl salicylate or methylsalicylate).mp

31 nabumetone.mp. or Nabumetone/

32 naproxen.mp. or Naproxen/

33 nimesulide.mp.

34 oxaprozin.mp. or Oxaprozin/

35 phenylbutazone.mp. or Phenylbutazone/

36 piroxicam.mp. or Piroxicam/

37 salicylate.mp. or Salicylates/

38 sulindac.mp. or Sulindac/

39 tenoxicam.mp.

40 tolmetin.mp. or Tolmetin/

41 tiaprofenic acid.mp.

42 niflumic acid.mp. or Niflumic Acid/

43 acetylsalicyclic acid.mp.

44 morniflumate.mp.

4512 or 13 or 14 or 15 or 16 or 17 or 18 or 19 or 20 or 21 or 22 or 23 or 24 or 25 or 26 or 27 or 28 or 29 or 30 or 31 or 32 or 33 or 34 or 35 or 36 or 37 or 38 or 39 or 40 or 41 or 42 or 43 or 44

4611 and 45

This is the search strategy for MEDLINE (OVID). We will combine it with the Cochrane Highly Sensitive Search Strategy for randomised trials: sensitivity and precision-maximising version (2008 revision) (Lefebvre 2019), to identify reports of randomised controlled trials. 
We will combine this search strategy with the Canadian Agency for Drugs and Technologies in Health (CADTH): MEDLINE adverse effects filter and/or with the BMJ Clinical Evidence: MEDLINE adverse effects strategy (both reported in Farrah et al 2016), to identify nonrandomized reports of adverse events.

The MEDLINE (OVID) strategy will be modified as needed for other electronic databases. All search strategies will be reported in the final version of the review.

\section{HISTORY}

Protocol first published: Issue 8, 2020

\section{CONTRIBUTIONS OF AUTHORS}

Roderick P Venekamp: scoped, designed, and drafted the protocol with the help of the other authors.

Rick T van Uum: commented on the draft protocol and agreed upon the final version.

Menelaos Konstantinidis: commented on the draft protocol and agreed upon the final version.

Vittoria Lutje: developed the search strategy, commented on the draft protocol, and agreed upon the final version.

Anne GM Schilder: commented on the draft protocol and agreed upon the final version.

Alastair D Hay: commented on the draft protocol and agreed upon the final version.

Roger AMJ Damoiseaux: commented on the draft protocol and agreed upon the final version.

Paul Little: scoped and designed the protocol, commented on the draft protocol, and agreed upon the final version.

\section{DECLARATIONS OF INTEREST}

Roderick P Venekamp: Roderick Venekamp is an Editor for Cochrane Acute Respiratory Infections and Cochrane ENT, but had no role in the editorial process for this review.

Rick T van Uum: none known.

Menelaos Konstantinidis: Statistical Editor for Cochrane Acute Respiratory Infections group, but had no role in the editorial process for this review.

Vittoria Lutje: Vittoria Lutje works as an independent consultant conducting literature searches for various research groups. None of them has any potential relevance to the submitted work.

Anne GM Schilder: the author's evidENT team at the UCL Ear Institute is supported by funding from the National Institute of Health Research (NIHR) and EU Horizon2020 for projects and fellowships. Prof Schilder is National Lead of the NIHR Clinical Research Network ENT Specialty, Surgical Specialty Lead for ENT for the Royal College of Surgeons of England's Clinical Research Initiative, and joint Co-ordinating Editor of Cochrane ENT.

Alastair D Hay: I sit on the National Institute for Health and Care Excellence (NICE) managing common infections guideline committee and am working on a Pfizer-funded study to conduct a community-based study to evaluate the burden of some vaccine-preventable infections not currently in the UK vaccine schedule.

Roger AMJ Damoiseaux: none known.

Paul Little: a study that I was $\mathrm{Cl}$ for had placebo and probiotic tablets given to us (the study was on sore throat) by a commercial company who makes probiotic (Cultech), and we have a very small grant for investigating the SSK probiotic for sore throat and the probiotic is being supplied by the company (Blis). Both of these are only very indirectly related (they do not relate to the use of NSAIDs).

The authors declare that no funding or support was received for this systematic review. 